\title{
FUNÇÃO PULMONAR NA EVOLUÇĀO DE 35 DOENTES COM PARACOCCIDIOIDOMICOSE
}

\author{
E. P. Caypos (1) \& A. J. M. Cataneo (2)
}

\section{R E S U M O}

Lesões pulmonares observadas na paracoccidioidomicose (pbmicose) pela radiologia foram designadas: leve, moderada e grave de acordo com critério estabelecido pelos autores. Lesões infiltrativas intersticiais bilaterais nódulo fibrolineares e cotonosas foram identificadas respectivamente em 34 e em 23 doentes. Formas leve, moderada e grave assinaladas respectivamente em 610 e 19 mostraram à análise radiológica evolutiva melhora em 2, piora em 15 e manutenção do padrão da lesão em 18 doentes.

Testes de função pulmonar realizados nos doentes durante o retorno ambulatorial evidenciaram: $12 \mathrm{com}$ padrão espirográfico normal, 20 obstrutivos e 3 mistos; 34 doentes estavam hiperventilando e todos apresentaram aumento da diferença alvéolo arterial.

Os resultados obtidos permitiram supor que a fibrose residual descrita nos padrões radiológicos; manutençăo e piora de 33 deles aliada à doença obstrutiva crônica verificada pelas provas de função pulmonar constituíram subsídios para o desenvolvimento do Cor pulmonale assinalado

UNITERMOS: Paracoccidioidomicose - Função pulmonar.

\section{INT R O D U C Ã O}

A pbmicose, causada pelo P. brasiliensis, é doença endêmica na América Tropical. O fungo atinge os pulmões pela via inalatória onde estabelece o complexo primário, semelhante ao da tuberculose, que evolui para cura ou disseminação $1,3,4,7,14$.

O envolvimento pulmonar da doença foi classicamente estudado por LrMA em 1952. O comprometimento pulmonar isolado ou não da pbmicose tem sido assinalado por diversos autores $3,4,7,13,16$. Alguns investigadores observaram lesões fibrocicatriciais pulmonares evolutivas na pbmicose $1,4,6,9,10,11$.
Testes de função pulmonar evidenciaram insuficiência ventilatória obstrutiva na maioria dos doentes blastomicóticos 1,4,6,9,10,11.

Lesões pulmonares residuais têm merecido nossa atenção no seguimento dos doentes, sendo assim, resolveu-se avaliar a função pulmo. nar de 35 pacientes acompanhados no Ambulatório de Moléstias Infecciosas da Faculdade de Medicina de Botucatu.

\section{MATERIAL E METODOS}

\section{Doentes Estudados}

(1) Prof. Adjunto do Departamento de Moléstias Infecciosas e Parasitárias - Dermatologia e Radiologia - Faculdade de Medicina de Botucatu - UNESP - 18600 Botucatu, S. Paulo, Brasil.

(2) Prof. Assistente Doutior - Disciplina de Cirurgia Toracica - Departamento de Cirurgia e Ortopedia da Faculdadia de Medicina de Botucatu - UNESP. 
CAMPOS, E. P. \& CATANEO, A. J. M. - Função pulmonar na pyolução de 35 doentes com paracoccidioidomicose. Rev. Inst. Med. trop. São Paulo, 28:330-336, 1986.

Estudaram-se 35 doentes com pbmicose pulmonar - isolada ou associada, tratados pela Anfotericina B $(1 \mathrm{mg} / \mathrm{kg}$ em S.G. a $5 \% \mathrm{em}$ dias alternados). Os doentes foram distribuídos de acordo com a faixa etária, sexo, raça, profissão, tipo de lavoura, procedência, antecedentes e duração da doença. As formas $e$ as manffestaçôes clínicas foram assinaladas. Comparou-se a pressão arterial antes com a da pós terapia tardia.

\section{Avaliação Radiológica}

Estudaram-se as imagens radiológicas pulmonares evolutivas e classificaram-nas em leve, moderada e grave. Designou-se lesão leve ao conjunto de infiltrados intersticiais nódulo fibrolineares bilaterais ou não, pouco extensos, com discreto enfisema ou sem ele, alargamen. to ou retração do hilo e do ângulo da carina $e$ arco da pulmonar normal. Denominou-se lesão moderada aquela que apresentasse infiltra. dos intersticiais nódulo fibrolineares bilaterais, floculosos, trabeculares ou não, calcificados, com enfisema de bases pulmonares, modificaçăo do hilo e do ângulo da carina e sinais indiretos de hipertensão pulmonar. Designouse lesão grave à associação de imagens radiológicas exsudativas bilaterais fibronodulares, floconosas, supurativas, trabeculares, miliarizadas, cavitações, sinais inđiretos de hipertensão pulmonar, mođificação do hilo e do ângulo da carina e enfisema. Os critérios radiológicos, eletrocardiográficos e clínico-evolutivos ca. racterizaram a hipertensāo puimonar e ou Cor pulmonale.

\section{Avaliação Laboratorial}

O diagnóstico etiológico foi estabelecido pela micologia direta e/ou biópsia e pelo exa. me otorrinolaringológico (O.R.L.). As alteracổes laboratoriais durante e após a terapia foram assinaladas. $O$ teste de Thorn e o traçado eletrocardiográfico foram estudados. Avaliou-se- perfil laboratorial evolutivo. As reações adversas tardias foram registradas. Realizouse o teste da paracoccidioiđina em 18 doentes. As reações sorológicas: fixação do complemen. to (F.C.), precipitação em tubos (p.p.), imunofluorescência indireta (I.I.) e imunodífusão em gel (I.D.) foram analisadas.

\section{Critério de Gura}

A remissão clínica foi caracterizada pela ausência de manifestações clínicas e pela negativação sorológica há mais de um ano de seguimento. Derivados sulfonamídicos foram utilizados na fase de consolidação da remissāoclínica.

\section{Estudo da Função Pulmonar}

Testes de função pulmonar foram realiza. dos no retorno ambulatorial.

Estudaram-se os seguintes atributos: volume corrente (VC), frequiência respiratória (f), volume minuto expirado (VE), volume do espaço morto fisiológico (VEM), ventilação alveolar minuto (VA), capacidade vital (CV), capacidade inspiratória (CI), volume de reserva ex piratória (VRE), capacidade vital forçada (CVF), volume expiratório forçado em um se. gundo/capacidade vital forçada (VEF1/CVF), fluxo expiratório forçado médio durante a me tade da CVF (FEF 25-75\%). Ventilação voluntária máxima (VVM), pH, pressão parcial de oxigênio $(\mathrm{PaO} 2)$ e pressão parcial de gás carbônico ( $\mathrm{PaCO} 2$ ) no sangue arterial com o paciente respirando ar ambiente, e a diferença alvéolo arterial das pressões parciais de oxi. gênio $\mathrm{P}(\mathrm{A}-\mathrm{a}) \mathrm{O}_{2}$ respirando $\mathrm{O}_{2}$ a $100 \%$.

A determinação dos atributos foi realiza. da conforme segue: VC, f e VE, CV, CI e VRE obtidos em gasōmetro Collins com capacidade para 120 litros. O volume do espaço morto foi calculado pela equação de Bohr modificada:

$$
\mathrm{VEM}=\frac{\mathrm{PaCO} 2-\mathrm{PECO} 2}{\mathrm{PaCO} 2} \times \mathrm{VC}
$$

A CVF, VEF1/CVF, FEF $2575 \%$ e VVM foram obtidos em espirómetro Med.Graf 200

$\mathrm{O} \mathrm{pH}$, e gases sangiüineos foram determinados em hemogasômetro (Corning) mod. $165 / 2$.

Baseados em trabalhos de BALDWIN e col. $(1948)^{2}$ e MORRIS e col. (1971) ${ }^{\text {is }}$ os valores considerados normais foram:

1. VEF1/CVF em porcentagem maior que $70 \%$. 
CAMPOS, E. P. \& CATANEO, A. J. M. - Funģão pulmonar na evoluçāo de 35 doentes com paracoccidioiơomicose. Rev. lnst. Med. trop. São Paulo, 28:330-336, 1986.

2. FEF $25-75 \%$ relativo ao valor predito maior que $70 \%$.

3. CV relativa ao valor predito mainr que $80 \%$.

A classificação dos pacientes obedeceu ao seguinte critério:

A. Padrāo ventilatório normal: itens 1,2 e 3 normais.

B. Padrão ventilatório obstrutivo: itens 1 e ou 2 abaixo do normal e item 3 normal.

C. Padrão ventilatório restritivo: itens 1 e 2 normais $\mathrm{e}$ item 3 abaixo do normal.

D. Padrão ventilatório misto: itens 1 e ou 2 abaixo do normal e item 3 abaixo do normal.

\section{RESULTADOS}

\section{Doentes Estudados}

A faixa etária oscilou de 16 a 68 anos com predomínio do sexo masculino (34:1) e da raça branca sobre a negra (10:1). A lavoura foi a atividade profissional de 33 dos 35 doentes, sendo a de café a mais referida. Antecedentes tabagistas, etilistas, pulmonares e digestivos foram assinalados respectivamente em $31,23,18$ e 11 doentes. A maioria deles provém de cidades vizinhas de Botucatu e 26 doentes referiram inicio da doença há 5 ou mais anos.

Os locais de aparecimento da doença mais referidos foram tegumentar e respiratória respectivamente em 14 e 13 pacientes.

As formas clínicas de pbmicose fora mpulmonares associadas em 33 e isoladas em 2 doentes. As associações mais encontradas foram: tegumentar em 20 , tegumento linfática em 7. linfática em 4 e linfática supra-renal em 2 pacientes. Icterícia foi observada em 2 doentes na forma linfática pulmonar.

As manifestações clínicas mais assinaladas acham-se expressas na tabela I. Hipertensão arterial foi registrada em 7 na pré e em 8 na pós terapia antifungica tardia. $O$ exame O.R.L. revelou lesão compativel com pbmicose em 15 dos 18 doentes examinados.
T A B E L A I

Manifestaçòes clinicas de 35 doentes com paracoccidioidomicose

\begin{tabular}{|c|c|c|c|}
\hline \multicolumn{2}{|l|}{ Respiratórias } & \multicolumn{2}{|l|}{ Digestivas } \\
\hline$\ldots \ldots$ & 25 & Sintomas gerais .... & 08 \\
\hline Tosse $\ldots \ldots \ldots$ & 23 & Dor abdominal ... & 03 \\
\hline Cor pulmonale ..... & 06 & Disfagia $\ldots \ldots \ldots$ & 02 \\
\hline Rouquidâo $\ldots \ldots \ldots$ & 05 & Icterícia $\quad \ldots \ldots \ldots \ldots$ & $\$ 2$ \\
\hline Distonia $\quad . \ldots \ldots \ldots$ & 04 & & \\
\hline Hemoptise $\ldots \ldots \ldots$ & (2) & Outras & \\
\hline Dor torácica ..... & 01 & Tegumentar $\ldots \ldots \ldots$ & 14 \\
\hline & & Gânglios $\quad \ldots \ldots \ldots \ldots$ & 11 \\
\hline & & Febre $\quad \ldots \ldots \ldots \ldots \ldots$ & 05 \\
\hline & & Addison,$\ldots \ldots \ldots$ & 02 \\
\hline & & Osteoarticular ..... & 02 \\
\hline
\end{tabular}

\section{Avaliação Radiológica}

O estudo radiológico das lesōes pulmonares de 3 a 12 anos de evolução da doença evidenciou predominio de infiltrados intersticiais bilaterais nódulo fibrolineares em 34 e cotonosos em 23 doentes. A distribuição para hilar bilateral e enfisema foram assinalados respectivamente em 32 e 25 doentes. Sinais radiológicos evolutivos sugestivos de hiperten. são pulmonar foram observados em 8 doentes dos quais 6 apresentaram clínica de Cor pulmonale. Comprometimento do lobo superior foi observado em 17, dos quais 6 apresentaram associação com a tuberculose. Cavitação. abscesso e paracoccidioma foram observados respectivamente em 6,5 e 3 das radiografias. Modificações de hilo e da carina foram descritas em 28. As formas radiológicas designadas, leve, moderada e grave foram assinaladas res pectivamente em 6,10 e 19 doentes. A análise radiológica evolutiva evidenciou melhora em 2, piora em 15 e manutenção da lesão em 18 doentes.

\section{Avaliação Laboratorial}

A micologia e a histopatologia confirmaram o diagnóstico de pbmicose. respectivamente em 35 e 34 doentes. A citoinclusão do escarro contribuiu para o diagnóstico da doença

Reações adversas mais observadas durante e após a terapia pela Anfotericina $B$ foram. hipopotassemia em 10, hiperpotassemia em 6 nefrotoxicidade em 6 sendo 4 com clearance dinimuído, arritmia cardíaca em 3 e lesões do $3 .^{\circ}, 4 .^{4}$ e 6..$^{\circ}$ pares craneanos em 1 doente. Um 
CAMPOS, E. P. \& CATANEO, A. J. M. -- Função pulmonar na evolução de 35 doentes com paracoccidioidomicose. Rev. Inst. Med. trop. São Paulo, ช9.330 $\div 336,1986$.

doente recebeu manitol a $25 \%$ para corrigir o clearance de creatinina diminuído. 15 deles näo apresentaram reaçóes adversas ao antifüngico. Realizaram-se 18 testes de Thorn, sendo que 9 dos 11 positivos tornaram-se hiporeatores no final da terapia pela Anfotericina $B$. Doenca de Addison foi registrada em dois doentes durante o seguimento ambulatorial. A hiperpotassemia de 5,6 a 6,6 meq/1 foi observada nos hiporeatores ao teste de Thorn, pneumopatas graves, Cor pulmonale e em um doente com hipertensão arterial. Não se registrou reação tardia decorrente do uso de Anfotericina $\mathrm{B}$.

\section{Critério de Cura}

Houve remissão clínica em 10, melhora em 4 e não cura em 21 doentes. Treze pacientes apresentaram melhora sorológica durante a evolução. Não houve concorância do critério de melhora e de não cura clínica com o da sorologia. A paracoccidioidina foi positiva em 15 e negativa em 5 doentes. As reações sorológicas de I.D., F.C., I.I. e p.p. dos 10 doentes "curados" oscilaram respectivamente de negativo a $1 / 2 ; 5,8$ a 1,9 ; menor ou igual a $1 / 64$ e de $(+)$ positiva a $(-)$ negativa. Ve. rificou se recaida após 5 ou mais anos do seguimento em $71,4 \%$ dos doentes.

\section{O Estudo da Funçãa Pulmonar revelou:}

5.1. Padrão Espirográfico - Dos 35 pacientes, 12 apresentaram padrão espirográfico normal, 20 foram considerados obstrutivos puros e 3 mistos. Dos 20 obstrutivos, 14 apresentaram alteração de VEF1/CVF e F'EF 25 $75 \%$, enquanto que 6 somente tiveram alterações de FEF 25-75\%.

5.2. Ventilação alveolar e espaço morto - O espaço morto foi superior a $35 \%$ do volume corrente em 18 pacientes $(51 \%)$ sendo 12 obstrutivos, 3 mistos e 3 normais.

A ventilação alveolar minuto foi inferior ao predito (2 a 25 S.C.) somente em um paciente que apresentava padrão obstrutivo; todos os demais hiperventilavam com uma VAM até 3,5 vezes do valor predito.

5.3. Gases Sangiúneos - Somente um paciente do grupo misto apresentou a $\mathrm{PaCO} 2$ acima de $45 \mathrm{mmHg}$.
A PaO2 esteve abaixo de $80 \mathrm{mmHg}$ em 22 pacientes sench 7 normais, 12 obstrutivos e 3 mistos. A $\mathrm{P}$ (A-a) $\mathrm{O}_{2}$ foi sempre elevada variando de 121 a $336 \mathrm{mmHg}$.

\section{DISCUSSĀO}

O predomínio do sexo masculino sobre o feminino, a faixa etária de 16 a 68 anos e as lavouras de café e cana como atividade profissional, constituiram achađos clássicos semelhantes aos da literatura na doença de LutzSplendore-Almeida $1,4,7,8,12,16$. Os antecedentes encontrados demonstraram a precária condição social e a grande suscetibilidade à doença. O tabagismo favorecendo o enfisema, o álcool e a cirurgia digestiva pela alteração imunológica merecem menção como predisponentes importantes da doença. Pneumopatias prévias em 18 pacientes facilitaram a reativacão da pbmicose $4,5,12,13,16$. A regiāo de Botucatu constitui área propícia para a aquisição da pbmicose $3,4,7$

O início da doença há mais de 5 anos reforça o caráter evolutivo crônico assinalado pelos diversos autores $1,3,4,7,8,12,16$.

As frequientes associaçōes assinaladas às formas pulmonares e as lesōes cutâneas presentes em 27 doentes evidenciaram o caráter sistêmico da doença de Lutz, fato análogo ao descrito por outros autores $3,4,5,7,8,11$. As lesões tegumentares e as adenopatias reafirmaram a disseminação relatada na pbmicose.

A importância do aparelho respiratório na doença de Lutz ficou evidenciada pela dispnéia, tosse e presença de cor puimonale clínico em 6 doentes.

As demais manifestações clínicas assinala das acham-se de acordo com as citadas por outros autores $3,4,5,7,11,13,14,16$. A pressão arterial normal registrada à internação e durante o seguimento ambulatorial impediram de responsabilizar a terapia pela hipertensão arterial tardia.

O predomínio de infiltrado intersticial, nó dulo-fibrolinear bilateral foi observado no estudo radiológico evolutivo dos doentes com manutenção e piora do padrão da lesão pulmonar O grande número de pacienves com enfisema foi provavelmente devido à associação do ta- 
CAMPOS, E. P. \& CATANEO, A. J. M. - Função pulmonar na evolução de 35 doentes com paracoccldioldomicose. Rev. Inst. Med. trop. São Paulo, 28:330-336, 1986.

bagismo à pbmicose pulmonar. FIALHO ${ }^{8}$, em 1946 descreveu o eniisema como parte da lesão anaiomopatológica da pbmícose. LIMA ${ }^{12}$, em 1952, referiu áreas de enfisema individualizadas ou em bolhas gasosas disseminadas devido à pbmicose pulmonar. $\mathrm{O}$ tabagismo portanto agravaria a lesão pulmonar da pbmicose $1,5,6,11$.

A fibrose pulmonar residual pós terapia e o enfisema assinalados seriam responsáveis pelo aparecimento da Cor pulmonale em 6 doentes. A presença de lesão apical na pbmicose suscita a necessidade de exclusāo ou associação com tuberculose 3,4,13,14,16. Cavitações, abscessos e paracoccidioma podem ser observado à radiologia segundo relato de alguns investigadores $4,5,1,12,14,16$. Alterações hilares sugeriram processos inflamatórios dos gânglios dessa região $3,4,8,12,14$.

A micologia e a histopatologia aliadas à citoinclusâo contribuiram para o acurado diag. nótico da pbmicose.

Reações adversas assinaladas durante $\mathbf{e}$ após a terapia imeđiata pela Anfotericina $B$ coincidiram com as citadas pela literatura $4,7,16$. As lesões do $3 .^{\circ}, 4 .^{\circ}$ e $6 .^{\circ}$ pares craneanos se devem ao complexo doença-terapia antifúngica. A infusão intravenosa de Manitol para corre. ção do clearance de creatinina parece encontrar apoio na literatura médica ${ }^{4}$. A hiperpotassemia foi observada nas condições clínicas descritas por Campos e cols. (1984) 4 .

O teste de Thorn hiporeator em 11 dos quais 9 no final da terapia antifúngica sugeriu envolvimento prévio da adrenal ou ação fibrótica da droga sobre a glândula que contém o paracoccidioides $3,4,5,7,16$. A presença da doença de Addison em 2 indivíduos colaborou com as hipoteses acima emitidas.

o longo período de seguimento permitiu observar a baixa taxa de cura clínica desses pacientes. O critério clínico desempenhou papel importante para definir cura sorologica.

A dificuldade da relação clínica com os achados sorológicos ficou evidente no presente estudo. Portanto a melhora quanto à não cura clínica deveriam ser apenas definidas a longo prazo, atê que o perfil clínico evolutivo da doença fúngica evidenciasse sua verdadeira característica 4,7,16. As reaçōes sorológicas I.D. e F.C. mostraram se adequadas em 10 doentes com critérios clínicos evolutivo de remissāo da doença.

A positividade da paracoccidioidina indicou maior número de remissão clínica da doenca 7,16 .

A espirometria mostrou que a maioria dos pacientes blastomicóticos apresentou pađrão ventilatório obstrutivo (57\%), sendo o FEF 25-75\% o parâmetro mais sensivel de avaliação, pois apresentou-se reduzido em todos os casos, enquanto que o VEFI/CVF só em 14 casos. Os 3 pacientes do grupo misto também apresentaram redução do FEF $25 \cdot 75 \%$, enquanto que o VEF1/CVF estava diminuido apenas em dois deles.

A $\mathrm{PaCO} 2$ se manteve normal em $97 \%$ dos doentes, possivelmente, devido a hiperventilação alveolar, no entanto, a $\mathrm{PaO}$ se manteve baixa em todos do grupo misto, $60 \%$ dos obs trutivos e $58 \%$ dos normais. Verificou-se que todos tinham aumento da $P$ (A a) 02, o que permitiu concluir que mesmo os pacientes com padrão espirográfico normal possuiam alterações alvéolo capilares, devido à fibrose intersticial provocada pela blastomicose que levaria a uma desproporção entre ventilação e perfusão e a um bloqueio alvéolo capilar.

A desproporção entre a ventilação e a perfusão aliada ao bloqueio alvéolo-capilar seriam responsáveis pela dispnéia referida pelos doentes pbmicóticos.

O fato de 31 dos 35 pacientes serem fumantes permitiu nos supor que o padrăo ventilatorio observado fosse devido parcialmente ao tabagismo. Resultados esses semelhantes aos de CHIBANTE \& REGO, 1983; LEMLE e cols., 1983. Por outro lado, RATTO e cols., 1979, mostraram que de 14 pacientes pbmicóticos náo fumantes, somente 3 não apresentaram padrão obstrutivo. Fenômenos obstrutivos em $78 \%$ dos doentes tratados e ou com alterações da luz bronquiolar foram relatados por CHIBANTE \& REGO, 1983. Áreas de enfisema individualizadas assinaladas por LIMA em 1952, assim como lesōes destrutivas de bronquiolos e de pequenos brônquios intralobulares descritos por FIALHO em 1946 explicariam o padrão obstrutivo obtido nos testes de função pulmonar. 
CAMPOS, E. P. \& CATANEO, A. J. M. - Funçāo pulmonar na evolução de 35 doentes com paracoccidioidomicose. Rev. Inst. Med. trop. São Paulo, 28:330-336, 1986.

A ocorrência dos processos restritivos na pbmicose estaria ligada à terapia e ou/doenças eventualmente associadas à fibrose residual assinalada por diversos autores $1,4,6,9,10,11$. Em nosso meio a técnica de administração do fármaco talvez possa explicar a menor ocorrência de fenômenos restritivos do que a descrita por outros autores $1,9,10,11$.

A fibrose residual demonstrada pelos padrões radiológicos de manutenção e piora em 33 doentes assim como a doença obstrutiva crônica confirmada pelas provas de função pulmonar provavelmente constituíram subsídios básicos para que 6 deles desenvolvessem Cor pulmonale.

\section{SUMMARY}

Pulmonary function in follow-up of 35 patients with paracoccidioidomycosis (South American Blastomycosis)

Pulmonary lesions observed in pbmycosis were denominated: mild, moderate and severe according to the criteria established from radiolgical findings by the authors.

Bilateral nodulofibrolinear and cotton interstitials infiltrates were identified respectivelly on 34 and 23 patients. Later radiological analyses showed mild, moderate and severe pul monary lesions respectivilly in $6 ; 10$ and 19 evolving to improved in 2; worsened in 15 and maintained in 18 patients.

Normal spirographic test in 12; obstructive defect in 20; mixed in 3; hyperventilation in 34 and alveolar arterial difference increased in all patients.

Residual fibrosis observed in radiological maintained and worsened of 33 themes added to chronic obstructive pulmonary disease recognized by lung function tests both were able to explain the development of Cor pulmonale syndrome in 6 patients.

\section{REFERENCIAS BIBLIOGRAFICAS}

1. AFONSO, J. E.; NERY, L. E.; ROMALDINI, H.; BOGOSSIAN, M. \& RIBEIRO RATO, O. - Função puimonar na paracoccidioidomicose (blastomicose sul-americana). Rev. Inst. Med. trop. S. Paulo, 21: 269-280, 1979.
2. BALDWIN, E. F.; COURNAND, A. \& RICHARDS Jr. D. W. - Pulmonary insufficiency. I. Physiological classification, clinical methods of analysis, standart valvues in normal subjects. Medicine (Baltimore), 27: 243-278, 1948 .

3. BRAS, K. - Observaciones sobre Ia anatomía patologica, patogénesis y evolución de la paracodcidioidomicosis. Mycopathologia (Den Haag), 37: 119.138, 1969.

4. CAMPOS, E. P.; HETCH, M. H.; SARTOR, J. '\& FRAN CO, M. F. - Aspectos clínicos e sorológicos evolutivos de 47 doentes com paracoccidioidomicose tratados pela Anfotericina B. Rev. Inst. Med. trop. 'S. Paulo, 26: 212-217, 1984

5. CAMPOS, E. P.; CATANEO, A. J. M.; TORCHIO, L. N. \& PEROTTI, L. A. - Paracoccidioidomicose pulmonar agressiva, endotraqueite estenosante e cor pulmonale sub-agudo. Descriçăo de um caso. Rev. Inst. Med. trop. S. Paulo, 28: 185-189, 1986.

6. CHIBANTE, A. \& REGO A. P. - Paracoccidioidomicose pulmonar: fisiopatologia. Folha méd., 86 (1/2): 67-69, 1983.

7. DEL NEGRO, G.; LACAZ,C. S. \& FIORILLO, A. M. Paracoccidioidomicose. Blastomicose sul-americana. Sāo Paulo, Sarvier; EDUSP, 1982.

8. FIALHO, A. S. - Localização pulmonar da "Micose de Lutz". Anatomia patológica e patogenia. Importância de seu estudo na patologia pulmonar. Rio de Janeiro, 1946. (Tese)

9. LEMLE, A.; WANKE, B. \& MANDEL, B. M. - Pulmonary localization of paracoccidioidomycosis: Iung function studies before and after treatment. Rev. Inst. Med. trop. S. Paulo, 25: 73-78, 1983.

10. IEMMLE, A.; VIEIRA, L. O.; MILWARD, G. A. \& LISBOA MIRANDA, J. - Lung function studies in pulmonary South American blastomycosis, Correlation with clinical and roentgenologic findings. Amer. J. Med., 48: 434-472, 1970.

11. LEMYE, A.; WANKE, B.; MIRANDA, J. L.; KROPF, G. L.; MANDEL, M. B. \& MANDEL, S. -m Pulmonary function in paracoccidioidomycosis (South American Blastomycosis). An analysis of the obstructive defect Chest, 83: 827-828, 1983.

12. LIMA, F. X. P. - Contribuição ao estudo clínico : radiológico da blastomicose pulmonar. Săo Paulo, 1952 (Tese de Doutoramento - Faculdade de Medicina da Universiaade de São Paulo).

13. LONDERO, A. T. \& SEVERO, L. C. - The gamut of progressive pulmonary paracoccidioidomycosis. Myco pathologia (Den Faag), 75: 65-69, 1981.

14. Magaltaxes, A. - Paracoccidioidomicose (blastom) cose sul americana). Aspectos radiológicos. Rev. Hosp Clin. Fac. Med. S. Paulo, 35: 147-155, 1980. 
CAMPOS, E. P. \& CATANEO, A. J. M. - Função pulmonar na evolự̆a de 35 doentes com paracoccldioldomicose. Rev. Inst. Med. trop. Sāo Paulo, 28:330-336, 1986.

15. MORRIS, J. F.; KOSKI, A. C. \& JOENSON, L, C. Spirometric standards for healthy nonsmoking adults Amer. Rev. resp. Dis., 103: 57-67, 1971.

16. RESTREPO M., A. - Paracoccidioides braslliensis. In: MENDELL, G. L.; DOUGLAS Jr., R. G. \& BENETT,
J. E. - Principles and practice of infectious diseases 2nd. ed. New York, John Wiley, 1985. cap. 227, p. 1499-1502.

Recebido para publicaçäo em 12/11/1985. 